\title{
Research on the Digital Hydraulic Cylinder Nonlinear Robust Controlling Methods with Input Saturation
}

\author{
Pan Wei, Zhu Shijian, Xu Shijie \\ College of Power Engineering, Naval Univ. of Engineering, Wuhan, China \\ forever19860418@126.comzhushij1955@hotmail.comxsjie1986@163.com
}

Keywords: digital hydraulic cylinder; position tracking control; nonlinear model; input saturation.

\begin{abstract}
First, the nonlinear model of the digital hydraulic cylinder and the controller are constructed, then the nonlinear problem is translated into the solving of a linear matrix inequality. Finally the anti-windup compensator is designed. Simulation results show that the proposed controller and compensator guarantee the robustness and good dynamic behavior of the system.
\end{abstract}

\section{Introduction}

With the development of the digital hydraulic technique, different methods are applied in the controlling of the digital hydraulic cylinder; however, most of them are based on the linear model with an absence on the certification of the system's nonlinear element and stability under the input saturation. The present essay is to design a nonlinear controller and an anti-windup compensator, which not only guarantee the system's stability, but also fulfill the system's dynamic requirements.

\section{The Nonlinear Model of the Digital Hydraulic Cylinder with Uncertain Parameter}

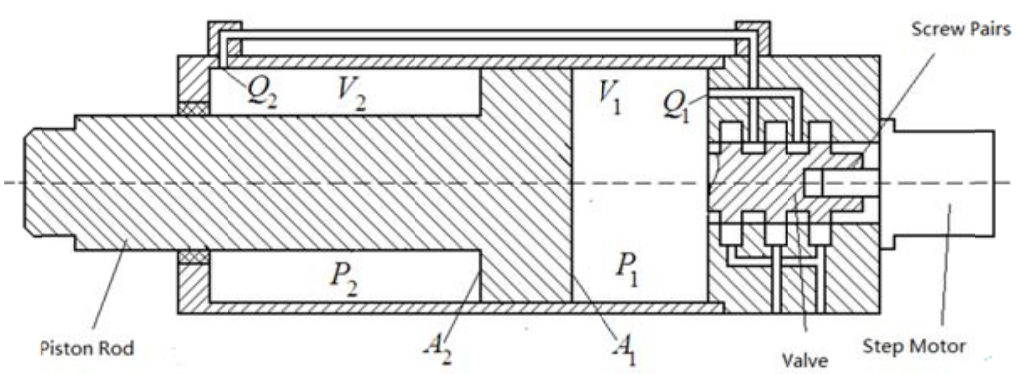

Fig 1 The inner structure of the digital hydraulic cylinder

The Fig. 1 is the inner structure of the digital hydraulic cylinder. $P_{1}$ and $P_{2}$ are the press of the non-rod chamber and the rod chamber, $Q_{1}$ and $Q_{2}$ are their flow, $V_{1}$ and $V_{2}$ are their volume, $A_{1}$ and $A_{2}$ are their effective area. The nonlinear model is as follows ${ }^{[1]}$. 


$$
\left\{\begin{array}{l}
\dot{x}_{1} \\
\dot{x}_{2} \\
\dot{x}_{3} \\
\dot{x}_{4}
\end{array}=\left\{\begin{array}{c}
x_{2} \\
\frac{A}{M}\left(x_{3}-E x_{4}\right)-\frac{k_{s}}{M} X_{1}-\frac{B_{p}}{M} X_{2}-F_{L} / M \\
-\frac{\beta_{e}}{V}\left[A x_{2}+C_{1}\left(x_{3}-X_{4}\right)\right]+u \frac{\beta_{e} R_{1}}{V_{1}} \begin{cases}\sqrt{P_{s}-X_{3}} & x_{v} \geq 0 \\
\sqrt{x_{3}-P_{0}} & x_{v}<0\end{cases} \\
\frac{\beta_{e}}{V_{2}}\left[A_{2} x_{2}+C_{1}\left(x_{3}-x_{4}\right)-C_{e} X_{4}\right]-u \frac{\beta_{e} R_{1}}{V_{2}} \begin{cases}\sqrt{x_{4}-P_{0}} & x_{v} \geq 0 \\
\sqrt{P_{s}-X_{4}} & x_{v}<0\end{cases}
\end{array}\right.\right.
$$

In Eq.(1): $x_{1}$ is the displacement of rod; $x_{2}$ is the speed of rod; $x_{3}=P_{1} ; x_{4}=P_{2} \cdot M$ is the equivalent quality; $k_{s}$ is the spring stiffness; $B_{p}$ is the damping coefficient; $\varepsilon=A_{2} / A_{1} ; \beta_{e}$ is the elastic modulus of oil volume; $V_{1}=V_{01}+A x_{1}, \quad V_{2}=V_{02}+A_{2} x_{1} ; V_{01}$ and $V_{02}$ are relatively the non-rod chamber and the rod chamber's initial volume; $R_{1}=\frac{C_{d} w_{f} t_{1}}{\sqrt{2 \rho} \pi}, C_{d}$ is the flow coefficient of valve port, $w_{f}=\pi d_{v}$ is the valve core area gradient, $d_{v}$ is the valve core diameter, $\rho$ is the density of hydraulic oil, $t_{1}$ is the lead of valve core; $P_{s}$ and $P_{0}$ are the oil pressure source and the Return pressure; $u$ is the input rotation angle of step motion; $F_{L}$ is the load force; $C_{i}$ is the internal leakage coefficient; $C_{e}$ is the external leakage coefficient. Let the input displacement signal be $r$, the tracking error signal be $e_{1}=x_{1}-r$, $e_{2}=x_{2}-\dot{r}=\dot{e}_{1}, e_{3}=\dot{x}_{2}-\ddot{r}=\dot{e}_{2}$.Let Eq. (1) Substituted:

$$
\left\{\begin{array}{l}
\dot{e}_{1} \\
\dot{e}_{2} \\
\dot{e}_{3}
\end{array}=\left\{\begin{array}{c}
e_{2} \\
e_{3} \\
\tau_{1} e_{1}+\tau_{2} e_{2}+\tau_{3} e_{3}+A_{1} / M\left(g_{3}-\varepsilon g_{4}\right) u+w
\end{array}\right.\right.
$$

Among them:

$$
\left\{\begin{array}{l}
\tau_{1}=-\beta_{e} C_{i} \frac{k_{s}}{M} \\
\tau_{2}=-\left[\frac{k_{s}}{M}+\frac{\beta_{e}}{M} A_{1}\left(\frac{A_{1}}{V_{1}}+\frac{\varepsilon A_{2}}{V_{2}}\right)+\frac{\beta_{e} B_{p}}{M} C_{i}\left(\frac{1}{V_{1}}+\frac{\varepsilon}{V_{2}}\right)\right] \\
\tau_{3}=-\left[\frac{B_{p}}{M}+\beta_{e} C_{i}\left(\frac{1}{V_{1}}+\frac{\varepsilon}{V_{2}}\right)\right] \\
g_{3}=\frac{\beta_{e} R_{1}}{V_{1}} \cdot \begin{cases}\sqrt{P_{s}-x_{3}} & x_{v} \geq 0 \\
\sqrt{x_{3}-P_{0}} & x_{v}<0\end{cases} \\
g_{4}=\frac{\beta_{e} R_{1}}{V_{2}} \cdot \begin{cases}\sqrt{x_{4}-P_{0}} & x_{v} \geq 0 \\
\sqrt{P_{s}-x_{4}} & x_{v}<0\end{cases} \\
w=\frac{A_{1}}{M} \beta_{e} X_{4}\left[\begin{array}{ll}
x_{4} C_{i}(1-\varepsilon) \\
V_{1}
\end{array}\left(\frac{1}{V_{1}}+\frac{\varepsilon}{V_{2}}\right)+x_{4} \frac{\varepsilon C_{e}}{V_{2}}-\left(\frac{A_{1}}{V_{1}}+\frac{\varepsilon A_{2}}{V_{2}}\right) \ddot{r}\right]-\frac{\beta_{e} C_{i}}{M}\left(\frac{1}{V_{1}}+\frac{\varepsilon}{V_{2}}\right)\left(k_{r} r+B_{p} \dot{r}+F_{L}+M \ddot{r}\right)
\end{array}\right.
$$

InEq.(2), take $w$ as disturbance, which is bounded. For the existence of input saturation, suppose $U$ 's value range is:

$$
|u| \leq u_{\max }
$$

In the hydraulic system,some complex elements caused the nominal value[2] inEq.(2). $M, \beta_{e}, C_{d}, R_{1}$ are the uncertain parameters considered in the present essay,they are expressed as:

$$
\begin{aligned}
& \frac{1}{M}=\frac{1}{\bar{M}}\left(1+\Delta_{1}\right) \\
& \beta_{e}=\bar{\beta}_{e}\left(1+\Delta_{2}\right) \\
& C_{d}=\bar{C}_{d}\left(1+\Delta_{3}\right) \\
& R_{1}=\bar{R}_{1}\left(1+\Delta_{3}\right), \bar{R}_{1}=\frac{\bar{C}_{d} w t_{1}}{\sqrt{2 \rho} \pi}
\end{aligned}
$$


The nominal parameters are respectively $\bar{M}, \bar{\beta}_{e}, \bar{C}_{d}, \bar{R}_{1} . \Delta_{1}, \Delta_{2}, \Delta_{3}$ describe respectively the uncertainty set of $M, \beta_{e}, C_{d}$ and $\left|\Delta_{1}\right| \leq a_{1},\left|\Delta_{2}\right| \leq a_{2},\left|\Delta_{3}\right| \leq a_{3}$. The certainty of $\tau_{1}, \tau_{2}, \tau_{3}, g_{3}$ and $g_{4}$ are $\overline{\tau_{1}} 、 \overline{\tau_{2}}$ 、 $\bar{\tau}_{3} 、 \bar{g}_{3} 、 \bar{g}_{4}$, their uncertainty are $\Delta \tau_{1}, \Delta \tau_{2}, \Delta \tau_{3}, \Delta g_{3}, \Delta g_{4}$, with $\left|\Delta \tau_{1}\right| \leq \tau_{m 1},\left|\Delta \tau_{2}\right| \leq \tau_{m 2},\left|\Delta \tau_{3}\right| \leq \tau_{m 3}$,Eq. (5) is as follows:

$$
\begin{aligned}
& \Delta \tau_{1}=\tau_{1}-\bar{\tau}_{1}, \Delta \tau_{2}=\tau_{2}-\bar{\tau}_{2}, \Delta \tau_{3}=\tau_{3}-\bar{\tau}_{3} \\
& \Delta g_{3}=g_{3}-\bar{g}_{3}, \Delta g_{4}=g_{4}-\bar{g}_{4}
\end{aligned}
$$

\section{The Design of the Digital Hydraulic Cylinder' Nonlinear Tracking Controller}

Eq. (2) could be constructedinto linear term The constructed controller is as follows:

$$
u=\frac{\bar{M} / A \cdot v}{\bar{g}_{3}-\varepsilon \bar{g}_{4}}
$$

In Eq. (6), $v$ is the unknown and is to meet the constraint $v_{\min } \leq v \leq v_{\max }, \quad v_{\max }=A / \bar{M}\left|\bar{g}_{3}-\varepsilon \bar{g}_{4}\right| u_{\max }$, $v_{\text {min }}=-A / \bar{M}\left|\bar{g}_{3}-\varepsilon \bar{g}_{4}\right| u_{\max }$ 。 Let Eq. (6) Substituted into Eq. (2) :

Among them:

$$
\begin{aligned}
& \dot{\hat{\boldsymbol{x}}}=(\boldsymbol{A}+\Delta \boldsymbol{A}) \hat{\boldsymbol{x}}+\left(\boldsymbol{B}_{2}+\Delta \boldsymbol{B}_{2}\right) \operatorname{sat}(v)+\hat{\boldsymbol{B}}_{1} w \\
& \boldsymbol{z}=\boldsymbol{C}_{2} \hat{\boldsymbol{x}}
\end{aligned}
$$

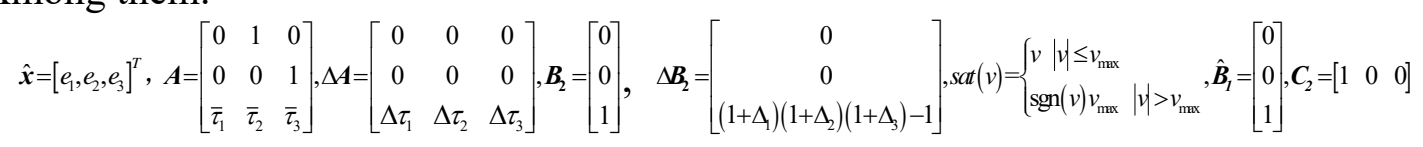

If $v$ could stabilize Eq.(7), $u$ could definitely stabilize Eq.(1). Therefore, the resolution is the solving to the parameter $v$ in Eq.(7). The construct controller $V$ is as follows:

$$
\left\{\begin{array}{l}
\dot{\boldsymbol{\eta}}=\boldsymbol{A}_{c} \boldsymbol{\eta}+\boldsymbol{B}_{c} \hat{\boldsymbol{x}}+E_{c}(v-\operatorname{sat}(v)) \\
v=C_{c} \boldsymbol{\eta}+\mathrm{D}_{c} \hat{\boldsymbol{x}}
\end{array}\right.
$$

As inEq. (8), $\boldsymbol{A}_{c}, \boldsymbol{B}_{c}, \boldsymbol{C}_{c}, \boldsymbol{D}_{c}$ are the unsolved matrix in the controller, the static compensation controller is $\boldsymbol{E}_{c}(v-s a t(v)), \quad \boldsymbol{E}_{c}$ is also the unsolved matrix. Firstly, the saturation of $v$ is not considered to the design of the controller, $A_{c}, B_{c}, C_{c}, D_{c}$ are obtained. In the next section, the compensator will be designed to the controller. ApplyEq.(8)toEq.(7), Eq.(9)is obtained:

$$
\left\{\begin{array}{l}
\dot{\xi}=A_{\xi} \xi+B_{\tilde{\xi} w} w \\
z=C_{\xi} \xi
\end{array}\right.
$$

Among them:

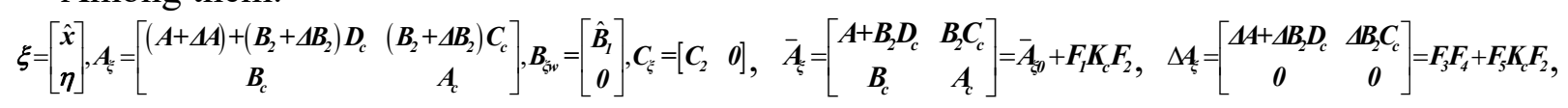

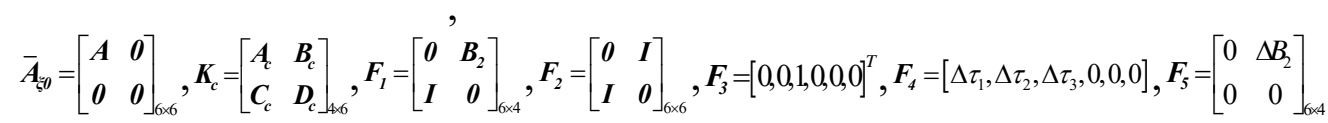

The necessary and sufficient condition which ensures that Eq.(5)is stabled and $L_{2}$ gain is smaller than $\gamma$ is ${ }^{[3-4]}$ :there exists Lyapunov function $V(\xi)>0, \dot{V}(\xi)<0$. Let $V(\xi)=\boldsymbol{\xi}^{T} \boldsymbol{P}_{l} \boldsymbol{\xi}, \quad \boldsymbol{P}_{l}$ is the positive definite symmetric matrix. If $\dot{V}(\xi)<0$ is tenable when $\boldsymbol{w}=0$, Eq.(10)is obtained:

$$
\left[\overline{\boldsymbol{A}}_{\xi_{0}}+\boldsymbol{F}_{1} \boldsymbol{K}_{c} \boldsymbol{F}_{2}+\boldsymbol{F}_{3} \boldsymbol{F}_{4}+\boldsymbol{F}_{5} \boldsymbol{K}_{c} \boldsymbol{F}_{2}\right]^{T} \boldsymbol{P}_{1}+\boldsymbol{P}_{1}\left[\overline{\boldsymbol{A}}_{\xi 0}+\boldsymbol{F}_{1} \boldsymbol{K}_{c} \boldsymbol{F}_{2}+\boldsymbol{F}_{3} \boldsymbol{F}_{4}+\boldsymbol{F}_{5} \boldsymbol{K}_{c} \boldsymbol{F}_{2}\right]<0
$$

If $\int_{0}^{t}\left(z^{T} z-\gamma^{2} \boldsymbol{w}^{T} \boldsymbol{w}\right) d \tau<0$ is wanted to be tenable when $\boldsymbol{w} \neq 0$, only $\boldsymbol{z}^{T} z-\gamma^{T} \boldsymbol{w}^{T} \boldsymbol{w}+\dot{V}(\xi)<0$ is needed. According to reference[5] lemma 2.2, the established condition to the above equation is:

$$
\begin{aligned}
& {\left[\bar{A}_{s 0}+\boldsymbol{F}_{1} \boldsymbol{K}_{c} \boldsymbol{F}_{2}+\boldsymbol{F}_{3} \boldsymbol{F}_{4}+\boldsymbol{F}_{5} \boldsymbol{K}_{c} \boldsymbol{F}_{2}\right]^{T} \boldsymbol{P}_{1}+\boldsymbol{P}_{1}\left[\bar{A}_{i 0}+\boldsymbol{F}_{1} \boldsymbol{K}_{c} \boldsymbol{F}_{2}+\boldsymbol{F}_{3} \boldsymbol{F}_{4}+\boldsymbol{F}_{5} \boldsymbol{K}_{c} \boldsymbol{F}_{2}\right]} \\
& +\boldsymbol{C}_{\xi}^{T} \boldsymbol{C}_{\xi}+\frac{1}{\gamma^{2}} \boldsymbol{P}_{l} \boldsymbol{B}_{\xi v}^{T} \boldsymbol{B}_{\xi v} \boldsymbol{P}_{I}<0
\end{aligned}
$$

Apply reference [5] lemma 2.1: there exists $\alpha>0$ which brings Eq.(12)into existence: 


$$
\begin{aligned}
& \overline{\boldsymbol{A}}_{\xi 0}^{T} \boldsymbol{P}_{I}+\boldsymbol{P}_{1} \overline{\boldsymbol{A}}_{\xi 0}+\boldsymbol{C}_{\xi}^{T} \boldsymbol{C}_{\xi}+\frac{1}{\gamma^{2}} \boldsymbol{P}_{I} \boldsymbol{B}_{\xi w}^{T} \boldsymbol{B}_{\xi w} \boldsymbol{P}_{I}+\alpha \boldsymbol{P}_{I} \boldsymbol{F}_{3} \boldsymbol{F}_{3}^{T} \boldsymbol{P}_{I}+\frac{1}{\alpha} \boldsymbol{F}_{6}^{T} \boldsymbol{F}_{6} \\
& +\boldsymbol{F}_{2}^{T} \boldsymbol{K}_{c}^{T}\left(\boldsymbol{F}_{1}+\boldsymbol{F}_{5}\right)^{T} \boldsymbol{P}_{I}+\boldsymbol{P}_{I}\left(\boldsymbol{F}_{1}+\boldsymbol{F}_{5}\right) \boldsymbol{K}_{c} \boldsymbol{F}_{2}<0
\end{aligned}
$$

InEq.(12): $\boldsymbol{F}_{6}=\left[F_{\tau}, 0,0,0\right]_{1 \times 6} ; \boldsymbol{F}_{\tau}=\left[\tau_{m 1}, \tau_{m 2}, \tau_{m 3}\right]$.If there exists scalar $\sigma$ to make Eq. (13) tenable by the applying of Finsler lemma ${ }^{[6]}$ :

$$
\begin{aligned}
& \boldsymbol{P}_{1}^{-1} \bar{A}_{\xi 0}^{T}+\overline{\boldsymbol{A}}_{\xi j} \boldsymbol{P}_{1}^{-1}+P_{1}^{-1} \boldsymbol{C}_{\xi}^{T} \boldsymbol{C}_{\xi} P_{1}^{-1}+\frac{1}{\gamma^{2}} \boldsymbol{B}_{\xi w}^{T} \boldsymbol{B}_{\xi w}+\alpha_{1} \boldsymbol{F}_{3} \boldsymbol{F}_{3}^{T}+\frac{1}{\alpha_{1}} \boldsymbol{P}_{1}^{-1} \boldsymbol{F}_{6}^{T} \boldsymbol{F}_{6} \boldsymbol{P}_{1}^{-1} \\
& -\sigma\left(\boldsymbol{F}_{1}+\boldsymbol{F}_{5}\right)\left(\boldsymbol{F}_{1}+\boldsymbol{F}_{5}\right)^{T}<0
\end{aligned}
$$

$\boldsymbol{K}_{c}=\frac{\sigma}{2}\left(\boldsymbol{F}_{1}+\boldsymbol{F}_{5}\right)^{T} \boldsymbol{P}_{1} \boldsymbol{F}_{2}$ is got.

$\left(\boldsymbol{F}_{1}+\boldsymbol{F}_{5}\right)\left(\boldsymbol{F}_{1}+\boldsymbol{F}_{5}\right)^{T} \geq \boldsymbol{F}_{7} \boldsymbol{F}_{7}^{T}$ and $\boldsymbol{F}_{7}=\left[\begin{array}{cc}0 & \left(1-a_{4}\right) \\ \boldsymbol{I} & 0\end{array}\right], \boldsymbol{B}_{2}, \boldsymbol{a}_{4}=\left(1+a_{1}\right)\left(1+a_{2}\right)\left(1+a_{3}\right)-1$. With the applying ofSchurcomplement lemma ${ }^{[4]}$, Eq. (13) could be expressed as linear matrixinequality :

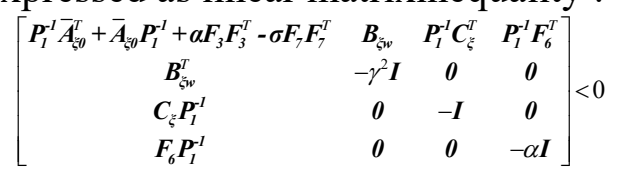

Take $\boldsymbol{P}_{1}^{-1}=\boldsymbol{X}=\left[\begin{array}{ll}\boldsymbol{X}_{I I} & \boldsymbol{X}_{12} \\ \boldsymbol{X}_{12} & \boldsymbol{X}_{13}\end{array}\right]$ and take the matrices into Eq.(14), Eq.(15) is obtained:

$$
\left[\begin{array}{ccccc}
\boldsymbol{\Sigma}_{1} & \boldsymbol{A} \boldsymbol{X}_{I 2} & \hat{\boldsymbol{B}}_{I} & \boldsymbol{X}_{I I} \boldsymbol{C}_{2}^{T} & \boldsymbol{X}_{I I} \boldsymbol{F}_{\tau}^{T} \\
\boldsymbol{X}_{I 2} \boldsymbol{A}^{T} & -\sigma \boldsymbol{I} & \boldsymbol{0} & \boldsymbol{X}_{I 2} \boldsymbol{C}_{2}^{T} & \boldsymbol{X}_{I 2} \boldsymbol{F}_{\tau}^{T} \\
\hat{\boldsymbol{B}}_{I}^{T} & \boldsymbol{0} & -\gamma^{2} \boldsymbol{I} & \boldsymbol{0} & \boldsymbol{0} \\
\boldsymbol{C}_{2} \boldsymbol{X}_{I I} & \boldsymbol{C}_{2} \boldsymbol{X}_{I 2} & \boldsymbol{0} & -\boldsymbol{I} & \boldsymbol{0} \\
\boldsymbol{F}_{\tau} \boldsymbol{X}_{I I} & \boldsymbol{F}_{\tau} \boldsymbol{X}_{I 2} & \boldsymbol{0} & \boldsymbol{0} & -\alpha \boldsymbol{I}
\end{array}\right]<0
$$

$\boldsymbol{\Sigma}_{1}=\boldsymbol{A} \boldsymbol{X}_{I I}+\boldsymbol{X}_{I I} \boldsymbol{A}^{T}+\alpha \boldsymbol{B}_{2} \boldsymbol{B}_{2}^{T}-\sigma\left(1-a_{4}\right)^{2} \boldsymbol{B}_{2} \boldsymbol{B}_{2}^{T}$ in Eq.(15).Eq.(15)is solved to get $P_{1}^{-1}$, and then $\boldsymbol{K}_{c}=-\frac{\sigma}{2} \boldsymbol{F}_{7}^{T} \boldsymbol{P}_{1} \boldsymbol{F}_{2}$ is got.So far, the system's controller is obtained.

\section{The Design to the Anti-windup Compensator}

Bring Eq.(8)into Eq.(7), Eq.(16) is got:

$$
\left\{\begin{array}{l}
\boldsymbol{\xi}=\boldsymbol{A}_{\xi} \xi+\boldsymbol{B}_{q} q+\boldsymbol{B}_{\xi w} w \\
\boldsymbol{v}=\boldsymbol{C}_{\boldsymbol{v}} \boldsymbol{\xi} \\
\boldsymbol{z}=\boldsymbol{C}_{\xi} \boldsymbol{\xi}
\end{array}\right.
$$

In Eq. (16): $q=v-\operatorname{sat}(v), \quad \boldsymbol{B}_{q}=\boldsymbol{B}_{q}+\boldsymbol{B}_{q 1} \boldsymbol{E}_{c}+\Delta \boldsymbol{B}_{q}, \boldsymbol{B}_{q}=\left[\begin{array}{c}-\boldsymbol{B}_{2} \\ \boldsymbol{0}\end{array}\right], \boldsymbol{B}_{q 1}=\left[\begin{array}{c}\boldsymbol{0} \\ \boldsymbol{I}_{663}\end{array}\right], \Delta \boldsymbol{B}_{q}=\left[\begin{array}{c}-\Delta \boldsymbol{B}_{2} \\ \boldsymbol{0}\end{array}\right], \Delta \boldsymbol{B}_{q} \Delta \boldsymbol{B}_{q}^{T} \leq\left[\begin{array}{cc}a_{4}^{2} \boldsymbol{B}_{2} \boldsymbol{B}_{2}^{T} & \boldsymbol{0} \\ \boldsymbol{0} & \boldsymbol{0}\end{array}\right]=\boldsymbol{F}_{8} \boldsymbol{F}_{8}^{T}, \boldsymbol{F}_{s}=\left[\begin{array}{c}a_{4} \boldsymbol{B}_{2} \\ \boldsymbol{0}\end{array}\right], \boldsymbol{C}_{v}=\left(\begin{array}{ll}\boldsymbol{D}_{c} & \boldsymbol{C}_{c}\end{array}\right)$

If there exists Lyapunov function $V_{1}(\xi)>0, \dot{V}_{1}(\xi)<0$ which makes $\int_{0}^{t}\left(z^{T} z-\gamma_{1}^{T} w^{T} w\right)<0$, Eq. (17) is stable and $L_{2}$ gain is smaller than $\gamma_{1}$. Among them $V_{1}(\xi)=\xi^{T} P_{\xi} \xi, P_{\xi}$ is the positive definite symmetric matrix. Derivative $V_{1}$ we can get that:

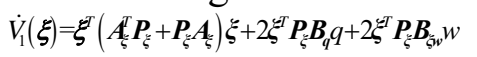

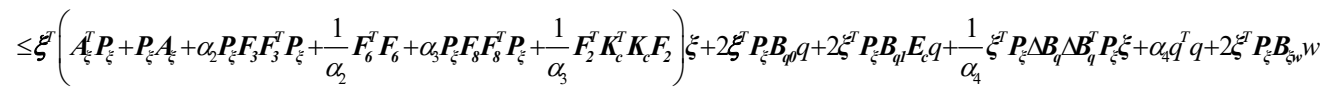

$$
\begin{aligned}
& \leq \xi^{T}\left[A_{\xi}^{T} \boldsymbol{P}_{\xi}+\boldsymbol{P}_{\xi} \boldsymbol{A}_{\xi}+\boldsymbol{P}_{\xi}\left(\alpha_{2} \boldsymbol{F}_{3} \boldsymbol{F}_{3}^{T}+\alpha_{3} \boldsymbol{F}_{\delta} \boldsymbol{F}_{s}^{T}+\frac{1}{\alpha_{4}} \boldsymbol{F}_{s} \boldsymbol{F}_{s}^{T}\right) \boldsymbol{P}_{\xi}+\frac{1}{\alpha_{2}} \boldsymbol{F}_{6}^{T} \boldsymbol{F}_{6}+\frac{1}{\alpha_{3}} \boldsymbol{F}_{2}^{T} \boldsymbol{K}_{c}^{T} \boldsymbol{K}_{c} \boldsymbol{F}_{2}\right] \boldsymbol{\xi}+2 \xi^{T} \boldsymbol{\xi}_{\xi} \boldsymbol{B}_{q 0} q+2 \xi^{T} \boldsymbol{\xi}_{\xi} \boldsymbol{B}_{q 1} \boldsymbol{E}_{c} q+2 \xi^{T} \boldsymbol{P}_{\xi} \boldsymbol{B}_{\xi v} w+\alpha_{4} q^{T} q
\end{aligned}
$$

Among them, $\alpha_{2}, \alpha_{3}, \alpha_{4}, \gamma_{1}$ are positive numbers 。Notice that positive number $\alpha_{5}$ could make $\alpha_{5} q^{T}(v-q)=\alpha_{5} q^{T}\left(C_{v} \xi-q\right) \geq 0$ tenable. If:

$\dot{V}(\xi)+z^{T} z-\gamma_{1}^{2} w^{T} w+2 a_{5} q^{T}(v-q)<0(18)$

The system could be stable. 


\section{Simulation Study}

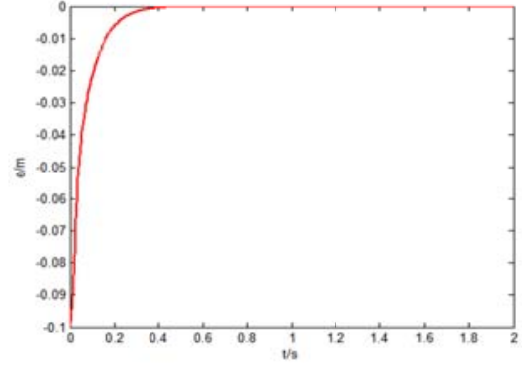

Fig. 2 Tracking error e

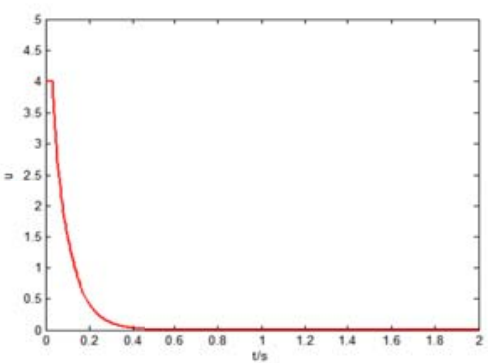

Fig. 3 Controller $\mathrm{u}$

The simulating of the designed controller is shown in Fig.2 and Fig.3.When the step signal is followed, the implying of the anti-windup controller could be faster in eliminating of the tracking error, withdrawing from the saturated zone and in the stabilization of the system.

\section{Conclusions}

The results indicate the anti-windup controller in the present design could track the input signal in a high speed, and have good robustness and adaptability for the parameter uncertainty.

\section{References}

[1] PENGLi-kun, XIAO Zhi-quan, XINGJi-feng, et al. Modeling and experiment study of a novel digital hydraulic 2-DOF motion platform[J]. Jixie Gongcheng Xuebao(Chinese Journal of Mechanical Engineering), 2011, 47(3): 159-165. (in Chinese)

[2] MILIĆ V, ŠITUM Ž, ESSERT M. Robust Ho position control synthesis of an electro-hydraulic servo system[J]. ISA transactions, 2010, 49(4): 535-542.

[3] LI Jian-xiong, FANG Yi-ming, SHI Sheng-li. Robust dynamic output-feedback control of hydraulic servo system with input saturation for rolling mill[J]. Control and Decision, 2013, 28(2): 211-216. (in Chinese)

[4] ZHOU K, DOYLE J C, GLOVER K. Robust and optimal control[M]. New Jersey: Prentice Hall, 1996.

[5] LIU B, TEO K, LIU X Z. Robust exponential stabilization for large-scale uncertain impulsive systems with coupling time-delays[J]. Nonlinear Analysis: Theory, Methods \& Applications, 2008, 68(5): 1169-1183.

[6] SKELTON R E, IWASAKI T, GRIGORIADIS K M. A unifiedalgebraic approach to linear control design[M]. London:Taylor \& Francis, 1998. 